$$
\iint_{S}\left\{\left(\frac{\partial u}{\partial r}\right)_{Q}-c\right\} d S_{Q}=-4 \pi c,
$$

while from (5), for $G \equiv 1$ and $H=w$, we have

$$
\iint_{S} \Delta_{2} w(Q) d S_{Q}=0
$$

THEOREM 3. If $(\partial u / \partial r)=c \neq 0$ on the open set $\Omega$ of the sphere $S$, and if $\Sigma$ is a domain containing $S$ and its interior, it is in no case possible to extend $u$ harmonically across $\Omega$ into the portion of $\Sigma$ exterior to $S$.

The University of California

\title{
ON THE CLASS OF METRICS DEFINING A METRISABLE SPACE*
}

\section{H. E. VAUGHAN}

Suppose we are given a metrisable $\dagger$ space $E$. Let $M$ be the class of all allowable metrics on $E$. Let $M_{b}, M_{c}, M_{B}$, and $M_{C}$ be, respectively, the classes of metrics in which the space is bounded, complete, totally bounded, and totally complete. The purpose of this note is to obtain systematically all possible theorems which state the equivalence of some topological property of $E$ (such as compactness, or separability) to the existence or non-existence of metrics having some of the above properties. An example is the well known theorem:

In order that $E$ be compact it is necessary and sufficient that it be complete in every allowable metric.

The problem may also be stated as follows: Using the four definitions as principles of classification and noting the inclusions $M_{b} \supset M_{B}$ $\supset M_{b} M_{C}$ and $M_{c} \supset M_{C} \supset M_{c} M_{B}$, we may represent $M$ as the sum of seven disjoint sets: (1) $M-M_{b}-M_{c}$, (2) $M_{b}-M_{B}-M_{b} M_{c}$, (3) $M_{c}-M_{C}$

* Presented to the Society, December 28, 1937.

$\dagger$ A topological space will be called metrisable if it is possible to define its continuity properties by means of a metric. Any metric which serves this purpose will be called allowable, and the space in conjunction with such a metric will be called a metric space. A metric space will be called bounded if there is a finite upper bound to the distance between any pair of its points. It will be called complete if every Cauchy sequence converges. It will be called totally bounded if it is, for every positive number $e$, the sum of a finite number of sets of diameter less than $e$. It will be called totally complete if every bounded set is compact. See C. Kuratowski, Topologie I, pp. 82, 87, 91, 196. 
$-M_{b} M_{c}$, (4) $M_{b} M_{c}-M_{c} M_{B}-M_{b} M_{C}$, (5) $M_{B}-M_{c} M_{B}$, (6) $M_{C}$ $-M_{b} M_{C}$, and (7) $M_{B} M_{C}$. These sets generate a Boolean algebra with $2^{7}$ elements. Any relation among these elements (which is sometimes, but not identically, satisfied) corresponds to some topological property of $E$, and the problem is to determine this correspondence.

Now, any such relation among the elements may be exhibited as a proposition constructed by the logical operations of conjunction, disjunction, and negation from the seven propositions obtained by setting each of the generating sets equal to the null set. Moreover, by the use of DeMorgan's Law, any such proposition may be expressed as a disjunction of conjunctions, the elements of the latter being chosen from the seven original propositions and their negations. The first step, then, is to obtain the topological equivalents of the $2^{14}$ conjunctions. Fortunately, only forty-five need be considered, the remainder falling into three classes: (a) those which are never satisfied, (b) those which are always satisfied, and (c) those of the form $P Q$ where $P$ is one of the forty-five and implies $Q$. It will be readily seen that when the topological equivalents of the above forty-five conjunctions are known, and when it is known that every other conjunction falls into one of the above three classes, it will be a rather easy matter to determine the topological equivalent of any given proposition of the type considered, or to determine whether or not such a proposition which is equivalent to a given property exists.

The topological part of the solution is contained in the following seven theorems and their converses. Proofs are given for the first three theorems. The proofs of the four remaining theorems, as well as of the seven converses, are obvious.

THEOREM 1. If $E$ is either bounded or complete in every allowable metric, then $E$ is compact.

Proof. Suppose that $E$ is not compact, and let $\left(a_{i}\right)$ be a divergent sequence of points of $E$. Let $R$ be a ray (half-line) with vertex 0 , and let $b_{2 n}$ be the point of $R$ whose distance from 0 is $1 / n$, and $b_{2 n-1}$ the point of $R$ whose distance from 0 is $n+1$. The subset of $E$ which consists of the points $a_{i}$ is closed and is homeomorphic to the subset of $R$ which consists of the points $b_{i}$. In fact the correspondence in which corresponding points have identical subscripts is a homeomorphism. Hence* there exists an allowable metric for $E$ such that this correspondence is an isometry. But in such a metric, $E$ is neither bounded nor complete, since the set whose elements are the points

* See F. Hausdorff, Erweiterung einer Homöomorphie, Fundamenta Mathematicae. vol. 16 (1930), pp. 352-360. 
$a_{2 i-1}$ is not bounded, while the sequence $\left(a_{2 i}\right)$ is a Cauchy sequence which does not converge to any point of $E$.

THEOREM 2. If $E$ is either totally bounded or complete in every allowable metric in which it is bounded, then $E$ is compact.

Proof. Suppose that $E$ is not compact, and let $\left(a_{i}\right)$ be a divergent sequence of points of $E$. Let $R^{\prime}$ be a metric space with points $b_{i}$, $(i=1,2, \cdots)$, and a metric defined as follows: The set consisting of the points $b_{2 n}$ is isometric to the similarly named point set in the preceding proof. The distance between any two points $b_{2 n-1}$ and $b_{2 m-1}$ is zero or one according as $n$ is or is not equal to $m$. The distance between $b_{2 n}$ and $b_{2 m-1}$, in either order, is $(2 n-1) / 2 n$. The triangle axiom is easily verified. As in the preceding proof there exists an allowable metric for $E$ such that the correspondence in which the point $a_{i}$ is associated with the point $b_{i}$ is an isometry. From this metric a new metric can be obtained, by the usual transformation $x^{\prime}=x /(1+x)$, in which the space is bounded. But in this metric, $E$ is neither totally bounded nor complete. For the set whose elements are the points $a_{2 i-1}$ is not totally bounded, while the sequence $\left(a_{2 i}\right)$ is a Cauchy sequence which does not converge to any point of $E$.

THEOREM 3. If $E$ allows a metric in which it is complete, and if $E$ is either totally complete or bounded in every such metric, then $E$ is compact.

Proof. Suppose that $E$ is not compact, and let $\left(a_{i}\right)$ be a divergent sequence of points of $E$. Choose a metric for $E$ as in the proof of Theorem 1. There exists* a metric in which $E$ is complete such that the distance in it of any two points of $E$ differs by at most one from the distance assigned to the two points by the metric of Theorem 1 . But in this (complete) metric, $E$ is neither totally complete nor bounded, for the set whose elements are the points $a_{2 i}$ has a diameter at most equal to two but is not compact, while the set whose elements are the points $a_{2 i-1}$ is not bounded.

TheOREM 4. If $E$ allows a metric in which it is bounded and complete, and if $E$ is either totally bounded or totally complete in at least one such metric, then $E$ is compact.

THEOREM 5. If $E$ allows a metric in which it is totally bounded, and if $E$ is complete in every such metric, then $E$ is compact.

THEOREM 6. If E allows a metric in which it is totally complete, and if $E$ is bounded in at least one such metric, then $E$ is compact.

* See C. Kuratowski, Topologie I, p. 200. 
THEOREM 7. If $E$ does not allow a metric in which it is both totally bounded and totally complete, then $E$ is not compact.

From these theorems together with the metric characterizations of separable spaces, ${ }^{*}$ of absolute $G_{\delta}$ 's, $\dagger$ and of locally compact, separable spaces $\ddagger$ it is now possible to determine the topological equivalents of the seven propositions mentioned above and of their negations.

$1 . \equiv . M-M_{b}-M_{c}=0 \quad . \equiv . E$ is compact.

$2 . \equiv . M_{b}-M_{B}-M_{b} M_{c}=0 \quad$. $\equiv . E$ is compact.

$3 . \equiv . M_{c}-M_{C}-M_{b} M_{c}=0 \quad . \equiv . E$ is compact or not an absolute $G_{\delta}$.

4. $\equiv . M_{b} M_{c}-M_{c} M_{B}-M_{b} M_{C}=0 . \equiv . E$ is compact or not an absolute $G_{\delta}$.

5. $\equiv . M_{B}-M_{c} M_{B}=0$

$6 . \equiv . M_{C}-M_{b} M_{C}=0$

7. $\equiv . M_{B} M_{C}=0$

$. \equiv . E$ is compact or not separable.

$. \equiv . E$ is compact or not separable or not locally compact.

It is seen that $1 . \equiv .2 . \equiv .-7$ and that $3 . \equiv .4$. Consequently, of the thirty-one conjunctions which contain two or more elements, only the eleven which can be built from $1,3,5,6$, and their negations need be listed. These are listed below, with their topological equivalents.

$3 \cdot 5 . \equiv . E$ is compact or not an absolute $G_{\delta}$ and is not separable.

$3 \cdot-1 . \equiv . E$ is not an absolute $G_{\delta}$.

$3 \cdot-5 . \equiv . E$ is separable and not an absolute $G_{\delta}$.

$5 \cdot-1 . \equiv . E$ is not separable.

$5 \cdot-3 . \equiv . E$ is an absolute $G_{\delta}$ and is not separable.

$6 \cdot-1 . \equiv . E$ is not separable or not locally compact.

$6 \cdot-3 . \equiv . E$ is an absolute $G_{\delta}$ and is either not separable or not locally compact.

$6 \cdot-5 . \equiv . E$ is separable and not locally compact.

$-3 \cdot-5 . \equiv . E$ is separable and an absolute $G_{\delta}$ and is not compact.

$3 \cdot 5 \cdot-1 . \equiv . E$ is not an absolute $G_{\delta}$ and is not separable.

$6 \cdot-3 \cdot-5 . \equiv . E$ is separable and an absolute $G_{\delta}$ and is not locally compact.

The completion of this list makes it easily possible to translate any proposition of the class considered into more familiar topological

* See Kuratowski, loc. cit., p. 91.

$\dagger$ See Kuratowski, loc. cit., p. 215.

$\ddagger$ See H. E. Vaughan, On locally compact metrisable spaces, this Bulletin, vol. 43 (1937), pp. 532-535. 
terms. The inverse problem of determining a proposition equivalent to a given topological condition, or showing that one does not exist, may be solved as follows: It will be seen that every condition which appears on the right side of one of the above equivalences is constructed, by means of the three elementary logical operations, from the four propositions:

\section{$A: E$ is compact.}

$B: E$ is locally compact and separable.

$C: E$ is separable.

$D: E$ is an absolute $G_{\delta}$.

Furthermore, these four may themselves be obtained from the conditions listed by means of the logical operations. It follows that the topological conditions which may be stated in terms of the existence or non-existence of metrics of the kinds considered are exactly those which can be obtained from the above four by conjunction, disjunction, and negation. To save space only the fourteen conditions involving $A, B$, and $C$, but not $D$, will be stated. The verification, by enumeration, of the fact that these are all offers no difficulty.

\section{$A: E$ is compact.}

$B: E$ is locally compact and separable.

$C: E$ is separable.

$-A: E$ is not compact.

$-B$ : $E$ is not locally compact or not separable.

$-C: E$ is not separable.

$B \cdot-A: E$ is locally compact and separable and not compact.

$C \cdot-A: E$ is separable and not compact.

$C \cdot-B: E$ is separable and not locally compact.

$A+-B: E$ is compact or not locally compact or not separable.

$A+-C: E$ is compact or not separable.

$A+C \cdot-B: E$ is compact or separable and not locally compact.

$B+-C: E$ is locally compact or not separable.

$-C+B \cdot-A: E$ is locally compact and not compact or not separable.

The proposed problem may now be considered as solved since any equivalence of the kind described in the first paragraph can now be constructed easily, starting with either the metric or the topological condition. It is now also possible to make statements like the following: There is no such equivalence in which the topological condition is local compactness. For the condition " $E$ is locally compact" does not appear in the preceding list.

\section{UNIVERSITY OF ILLINOIS}

